\title{
Experimental study on the effect of ultrasonic treatment on scaling process
}

\author{
Zepeng Wang ${ }^{1,}$, , Yongchang Chen ${ }^{1, b}$ \\ Key Laboratory of heat transfer enhancement and energy conservation of Ministry of Education, \\ Beijing University of Technology, BeiJing, China. \\ a17801070238@163.com, bchenyongchang@bjut.edu.cn.
}

\begin{abstract}
Keywords: ultrasound, power, temperature, scale inhibition rate.
Abstract. In this paper, the influence of circulating cooling water temperature and ultrasonic treatment on scaling process is investigated by using the experimental platform of fouling dynamic monitoring, and the change rule of fouling thermal resistance and scale inhibition rate are defined. In the process of experiment, ultrasonic frequencies is $20.7 \mathrm{KHZ}$, and the power changes from 0 to 100 $\mathrm{W}$. The experimental result shows that, under the condition of higher water temperature, the scaling ability of circulating water is enhanced, and the fouling thermal resistance is higher. Under the ultrasonic treatment, fouling thermal resistance decreases, and the scale inhibition rate increases with the increase of ultrasonic power, and the scale inhibition rate is up to $36.8 \%$.
\end{abstract}

\section{Introduction}

In industrial production, fouling can cause a decline in the heat transfer performance of heat exchange equipment, and make flow resistance increased. This not only causes great waste of resource, also may cause potential safety trouble due to heat transfer slowly, which affects the production efficiency seriously. Governance in the fouling, ultrasonic scale inhibition technology can work on-line continuously, automatically, reliably and have no influence on environmental. A lot of researches are reported about ultrasonic scale inhibition technology in recent years. Liang [1] showed that the scale inhibition rate is more than $85 \%$ when ultrasonic treatment on the water with frequency of $20 \mathrm{KHZ}$ and power of $100 \mathrm{~W}$. Huang et al. [2] used the simulation device of circulating water, under the same ultrasonic frequency, the scale inhibition effect with different ultrasonic power and velocity is investigated. The result shows that the larger ultrasonic power and the flow is, the better scale inhibition effect is. Zhang et al. [3] studied the change rule of ultrasonic sound intensity with temperature. The result shows that the ultrasonic attenuation decreases with the increase of temperature when the temperature is in the range of 25 to 55 centigrade, and the ultrasonic attenuation increases with the increase of temperature in the range of 55 to 75 centigrade. Sun [4] studied the effect of ultrasonic parameters on the scale inhibition effect. The research shows that when the power is increased, the scale inhibition rate will increase. With the higher ultrasonic frequency, the fluid flow rate have greater effect on ultrasonic scale inhibition effect. Zhao [5] used the experimental platform of fouling dynamic monitoring, it is found that the ultrasonic water treatment has a more obvious scale inhibition effect, and the frequency of $28 \mathrm{KHZ}$ ultrasonic scale inhibition effect is better than the frequency of 40KHZ. Luai M. Al-Hadhrami [6] studied the effect of flow rate on the fouling thermal resistance of twisted tube. It was found that when the flow rate was $1-2 \mathrm{~m} / \mathrm{s}$ the fouling thermal resistance was significantly less than that of $0.5 \mathrm{~m} / \mathrm{s}$. M. Legay [7] studied on the scale inhibition performance of ultrasonic frequency on heat exchanger. It was found that frequency is decreased from 1.6MHZ to $20 \mathrm{KHZ}$, the heat transfer coefficient increases two times.

However, due to the different conditions of the current research and the actual application of complex diversity, we are lack of relevant data as a basis in the actual application. In addition, ultrasonic scale inhibition mechanism is still not clear, resulting in ultrasonic scale inhibition technology is still not applied effectively. In this research, the scaling process of heat exchanger in industrial production is simulated by preparing artificial hard water, and the effect of temperature and ultrasonic treatment on scale inhibition performance is explored, so as to provide a reliable guarantee for the practical application. 


\section{Experimental device and method}

The experiment is carried out on the fouling thermal resistance dynamic monitoring device [9], which is composed of the experimental section, the cooling water loop, the hot water loop, the refrigeration system and the measurement and control system. The experimental section is a counter current tube heat exchanger. The inner tube is made of copper tube with smooth surface, and the outer tube is made of 304 stainless steel. The hot water (pure water) goes in the copper pipe, cooling water (artificial hard water) goes around the copper pipe, and hot water transfers heat to cooling water. The cooling water scales on the outer surface of the copper pipe, and the middle part of the outer tube is made of transparent glass tube to achieve the visualization of the scaling process. An ultrasonic scale inhibitor device for water treatment is installed on the cooling water loop, which is composed of an ultrasonic power supply and an ultrasonic transducer. The input voltage of the ultrasonic power supply is $220 \mathrm{~V}$, and the ultrasonic power can be adjusted continuously within $0-100 \mathrm{~W}$. The ultrasonic transducer is the key part of the ultrasonic scale inhibitor, which converts the high frequency electric oscillation to the mechanical vibration of the same frequency. In this experiment, the frequency of $20.7 \mathrm{KHZ}$ piezoelectric ceramic transducer is adopted. The measurement and control system is collected by data acquisition instrument automatically, and the temperature and pressure of the inlet and outlet of the heat exchange test section are respectively measured.

The artificial hard water is put into the cold water tank after the system is stable, which is made of pure $\mathrm{CaCl}_{2}$ and $\mathrm{NaHCO}_{3}$ with molar of 1:2. In the experiment, the heat transfer coefficient, fouling thermal resistance and scale inhibition rate can be calculated by using the experimental data. The main calculation formula is as follows:

Scale inhibition rate: $\eta_{f}=1-\frac{R_{\mathrm{a}}}{R_{f}}$

Where, $R_{f}$ is the copper tube fouling thermal resistance without ultrasound treatment, $\left(\mathrm{m}^{2} \cdot \mathrm{K} / \mathrm{W}\right)$, $R a$ is the copper tube fouling thermal resistance after ultrasound treatment, $\left(\mathrm{m}^{2} \cdot \mathrm{K} / \mathrm{W}\right)$.

\section{Experimental results and analysis}

The effect of ultrasonic power on fouling thermal resistance in the lower cooling water temperature. In the experiment, cooling water flow is maintained at $774 \mathrm{~L} / \mathrm{h}$, and hot water flow is $812 \mathrm{~L} / \mathrm{h}$. Initial hardness of cooling water is $500 \mathrm{mg} / \mathrm{L}$. The frequency of ultrasound is $20.7 \mathrm{KHZ}$, and the ultrasonic power is adjusted to $25 \mathrm{~W}, 50 \mathrm{~W}, 75 \mathrm{~W}$ and $100 \mathrm{~W}$ in turn. The hot water inlet temperature of the heat exchanger is maintained at $70^{\circ} \mathrm{C}$, and the cooling water inlet temperature is maintained at $22.5^{\circ} \mathrm{C}$. Each experiment lasts for 24 hours.

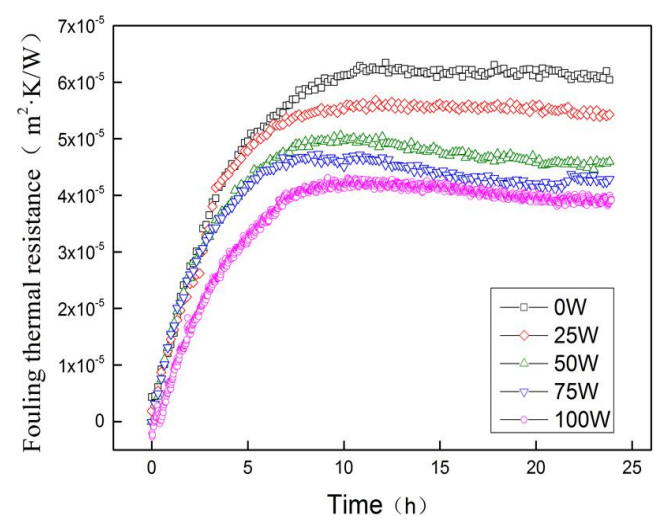

Fig.1 Ultrasonic effect on fouling thermal resistance $\left(22.5^{\circ} \mathrm{C}\right)$

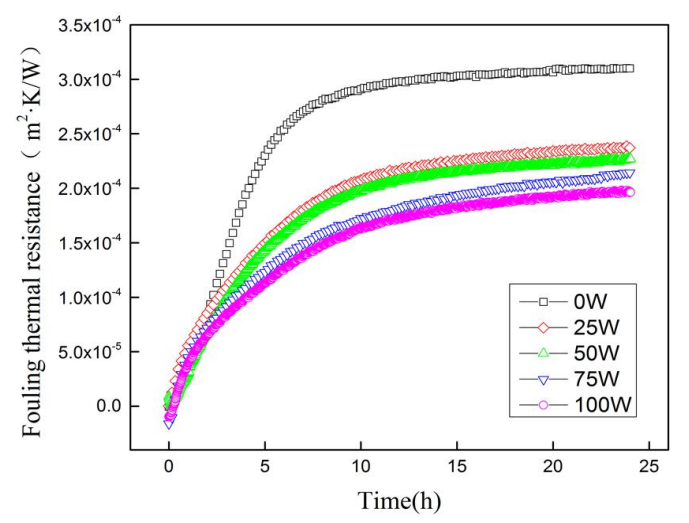

Fig.2 Ultrasonic effect on fouling thermal resistance $\left(44^{\circ} \mathrm{C}\right)$

Figure 1 shows whether there is or is not been treated by ultrasound, there is a sharp increase in fouling thermal resistance in the first five hours, and with the increase of ultrasonic power, the growth rate of fouling thermal resistance has been slow. The fouling thermal resistance is no longer changed significantly after ten hours or so. The maximum fouling thermal resistance without ultrasound is 
$6.03 \times 10^{-5} \mathrm{~m}^{2} \cdot \mathrm{K} / \mathrm{W}$, and the final fouling thermal resistance reduces from $2.37 \times 10^{-4} \mathrm{~m}^{2} \cdot \mathrm{K} / \mathrm{W}(25 \mathrm{~W})$ to $1.96 \times 10^{-4} \mathrm{~m}^{2} \cdot \mathrm{K} / \mathrm{W}(100 \mathrm{~W})$ with the increase of power. Thus, when the cooling water is in the lower temperature condition, and other conditions are the same, the greater the ultrasonic power is, the smaller the value of fouling thermal resistance can achieve, the better the ultrasonic scale inhibition effect is.

The effect of ultrasonic power on fouling thermal resistance in the higher cooling water temperature. In the experiment, the inlet temperature of cooling water is maintained at $44^{\circ} \mathrm{C}$, other conditions are same with the above part. Figure 2 shows that after ultrasound treatment, heat exchanger fouling growth rate was significantly lower than that without ultrasonic treatment when the growth rate and fouling thermal resistance stable time later than without ultrasonic. The maximum fouling thermal resistance without ultrasound is $3.10 \times 10^{-4} \mathrm{~m}^{2} \cdot \mathrm{K} / \mathrm{W}$, and the maximum fouling thermal resistance with ultrasonic treatment reduces from $2.37 \times 10^{-4} \mathrm{~m}^{2} \cdot \mathrm{K} / \mathrm{W}(25 \mathrm{~W})$ to $1.96 \times 10^{-4}$ $\mathrm{m}^{2} \cdot \mathrm{K} / \mathrm{W}(100 \mathrm{~W})$ with the increase of power. Thus, when the cooling water is in the higher temperature conditions, and other conditions are the same, the greater the ultrasonic power is, the smaller the value of fouling thermal resistance can achieve, the better the ultrasonic scale inhibition effect is.

Comprehensive figure 1 and figure 2 can be obtained, regardless of the higher temperature fluid or lower temperature fluid, the initial growth rate of fouling thermal resistance has been slow under been ultrasound treated. This is the process of fouling off the heat transfer surface in the form of fine particles, which is caused by cavitation bubble pulsation or collapse of ultrasonic cavitation effect, resulting in turbulence, circulation and the tangential stress, weakened the combination with force between the particle and neighboring particles. This weakened effect is enhanced with the coalescence of the particles of fouling, until the force exerted on the coalescence particles exceeds the binding force between the particles and the neighboring particles. In fact, it is the fouling particles off the heat transfer surface that runs through the whole process of fouling formation. Compared with the growth of fouling, the detachment of fouling showed the decrease of the fouling growth rate. Moreover, the higher temperature accelerates the fouling particles off the surface of the heat exchanger, resulting the stable time period of fouling thermal resistance later than without ultrasonic.

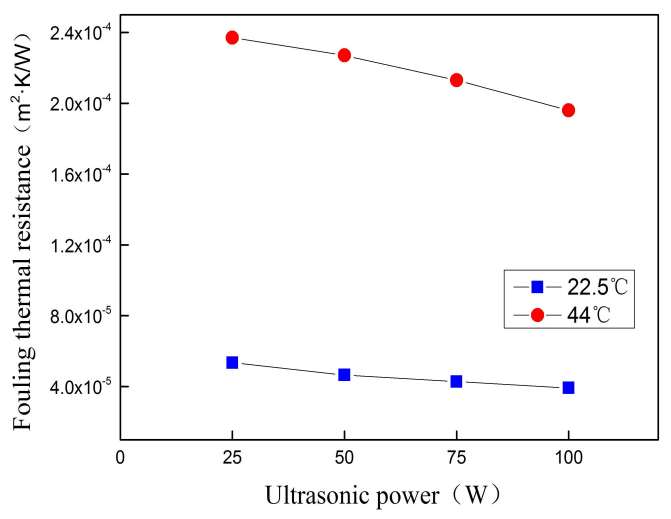

Fig. 3 Final fouling thermal resistance at different power

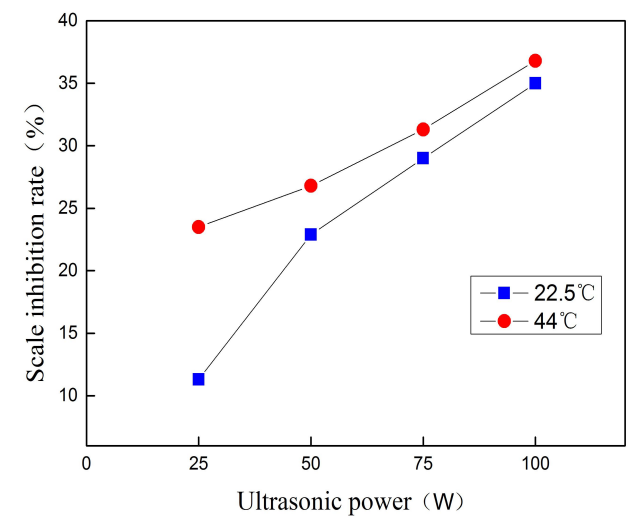

Fig.4 Effect of ultrasonic power on scale inhibition rate

The effect of ultrasonic power on scale inhibition performance. Figure 3 shows that different ultrasonic power correspond to the maximum fouling resistance in two different temperature of circulating cooling water. It can be found that with the increase of ultrasonic power from $25 \mathrm{~W}$ to $100 \mathrm{~W}$, the fouling thermal resistance gradually decreases. By the analysis, it is believed that the radiation of the ultrasound can cause the liquid medium to be processed to produce the cavitation effect. It causes the fouling particles to be crushed and suspended in the liquid medium, and it can also make the fouling layer broken and easy to fall off. At the same time, it can make the water splitting $\mathrm{OH}^{-}$ions that can form $\mathrm{Ca}(\mathrm{OH})^{+}$complexes with $\mathrm{Ca}^{2+}$ ions. Such complexes dissolved in water easily, reducing the formation of $\mathrm{CaCO}_{3}$ particles. However, the cavitation effect of the liquid medium need the sound pressure to reach cavitation threshold value. With the increase of ultrasonic power, ultrasonic cavitation enhancement, and heat surface fouling thermal resistance decreases, so 
we can get a better inhibition effect. Based on the above explanation, by the formula (1), the smaller the fouling thermal resistance is, the larger the scale inhibition rate is. Therefore, figure 4 shows that as the ultrasonic power increasing from $25 \mathrm{~W}$ to $100 \mathrm{~W}$, scale inhibition rate increased gradually.

The effect of temperature on scale inhibition performance. On the one hand, higher temperature not only reduces the solubility of $\mathrm{CaCO}_{3}$ in solution, resulting the $\mathrm{CaCO}_{3}$ crystal particles precipitate more, but also enhance $\mathrm{CaCO}_{3}$ self-crystallization in solution, making the $\mathrm{CaCO}_{3}$ particles suspended more in solution. That is to say, $\mathrm{CaCO}_{3}$ in the form of fouling in the pipeline supplies the chance on the surface of the heat exchanger, so the fouling resistance will become much larger. On the other hand, With the increase of temperature, the metastable aragonite form of $\mathrm{CaCO}_{3}$ have obvious precipitation, so the fouling particles is not easy to attach to wall and to be washed in liquid fluid easily. We can see from Figure 3, when the other conditions are the same, with the increase of temperature, the fouling thermal resistance increase gradually, which indicates that the rate of $\mathrm{CaCO}_{3}$ crystal particles depositing on the surface is far greater than the rate of $\mathrm{CaCO}_{3}$ particles off the surface, so that the rate of fouling resistance increases gradually.

\section{Conclusions}

In this paper, the influence of circulating cooling water temperature and ultrasonic treatment on scaling process is investigated by using the experimental platform of fouling dynamic monitoring, and the change rule of fouling thermal resistance and scale inhibition rate are defined. The experimental results show that, under the condition of higher water temperature, the scaling ability of circulating water is enhanced, and the fouling thermal resistance of circulating water is higher. Regardless of the higher temperature or lower temperature fluid, the fouling thermal resistance decreases by ultrasonic treatment. With the ultrasonic power from $25 \mathrm{~W}$ to $100 \mathrm{~W}$ in turn, the inhibition rate increased gradually, and the maximum value of inhibition rate is $36.8 \%$.

\section{Acknowledgements}

This work was financially supported by the Natural Science Foundation of China (10005014201201).

\section{References}

[1] Liang Chenghao, Bai Xinping. Chinese Water\&Waste Water. 2003 (S1): 64-66.

[2] Huang Leiuol, Zhang Mingduo, Niu Yong, et al. Journal of Shanxi Normal University (NATURAL SCIENCE EDITION). 2011(01): 38-40.

[3] Zhang Aiping, Hu Jianwen. Chemical machinery. 2011 (01): 38-40.

[4] Sun Xiaoqing. Experimental study on the operating parameters and the pipeline parameters of ultrasonic anti fouling flow liquid D. Shaanxi Normal University.

[5] Zhao Yang, Chen Yongchang, Meng Tao, et al. Journal of engineering thermal physics. 2013 (11): 2144-2146.

[6] Al-Hadhrami L M, Ahmad A, Al-Qahtani A. HEAT TRANSFER ENGINEERING. 2012, 33(12): 1024-1032.

[7] Legay M, Simony B, Boldo P, et al. Ultrasonics Sonochemistry. 2012, 19(6): 1194-1200.

[8] Quan Zhenhua, Wang Chunming, Li Bing, et al. water purification technology. 2007 (06): 30-33. 